\title{
Is Salvage of Recently Infected Breast Implant After Breast Augmentation or Reconstruction Possible? An Experimental Study
}

\author{
P. Castus ${ }^{1}$ - O. Heymans ${ }^{2}$ - P. Melin ${ }^{3}$ - L. Renwart ${ }^{2}$ - C. Henrist ${ }^{4}$ - E. Hayton ${ }^{5}$ • \\ S. Mordon ${ }^{6}$ F. M. Leclère ${ }^{6,7,8}$
}

Received: 15 September 2017/Accepted: 13 November 2017/Published online: 23 January 2018

(C) Springer Science+Business Media, LLC, part of Springer Nature and International Society of Aesthetic Plastic Surgery 2018

\begin{abstract}
Introduction The reinsertion of an infected implant when peri-prosthetic infection occurs early after breast augmentation or breast reconstruction remains controversial. In this experimental study, the authors tried to remove bacteria, and their biofilm, from the colonized surface of breast prostheses, without damaging their integrity.

Materials and Methods A total of 112 shell samples of silicone breast prostheses, smooth (SPSS) and textured (TPSS), were colonized by S. epidermidis (SE) or S. aureus (SA) strains, all able to produce biofilms. After 15 days, all
\end{abstract}

\section{F. M. Leclère \\ franckleclere@yahoo.fr}

1 Department of Plastic Surgery, Cliniques de l'Europe, Ste Elisabeth, Avenue de Fré 206, 1180 Brussels, Belgium

2 Department of Plastic and Maxillo-Facial Surgery, CHU Sart Tilman, 4000 Liège, Belgium

3 Department of Medical Microbiology, CHU Sart Tilman, 4000 Liège, Belgium

4 Department of Chemistry, Science Faculty, Domaine Universitaire du Sart Tilman B6, 4000 Liège, Belgium

5 Department of Infectious Diseases, Saint-Louis Hospital, 75010 Paris, France

6 INSERM, Univ. Lille, CHU Lille, U1189-ONCO-THAIImage Assisted Laser Therapy for Oncology, Lille, France

7 Department of Plastic and Transsexual Surgery, Burn Surgery, Hand Surgery, Centre of Expertise for Sex Reassignment Surgery, , CHU University Hospital of Bordeaux and University of Bordeaux, Centre FrançoisXavier-Michelet, Groupe Hospitalier Pellegrin, Place Amélie-Raba-Léon, 33076 Bordeaux, France

8 Department of Plastic Surgery, University of Poitiers, University Hospital CHU de Poitiers 2rue de la Milétrie, 86000 Poitiers, France the samples were removed from the contaminated culture broth and constituted 4 groups of 20 contaminated samples: SPSS/SE (group I), SPSS/SA (group II), TPSS/SE (group III), TPSS/SE (group IV). In another group_-group SEM-, 16 colonized samples were used for documentation with scanning electron microscopy (SEM). The remaining 16 samples were used to test the limits of detection of the sterility test. All samples of groups I-IV and 8 samples of group SEM were «washed » with a smooth brush in a povidone-iodine bath and rinsed with saline solution. A subset of the washed samples was sent for SEM and the others were immersed in sterile broth and were incubated at $35^{\circ} \mathrm{C}$ for 3 weeks (groups I-IV).

Results Fifteen days after contamination, all the samples in groups I-IV were colonized. In the SEM group, SEM images attested to the presence of bacteria in biofilm attached to the shells. After cleaning, SEM did not reveal any bacteria and there was no visible alteration in the outer structure of the shell. Sterility tests performed after decontamination in groups I-IV remained negative for all the samples.

Conclusion Breast prostheses recently contaminated with Staphylococci, frequently involved in peri-prosthetic breast implant infection and capable of producing biofilms, can be efficiently decontaminated by the procedure used in this study. Our decontamination procedure did not alter the surface structure of the prostheses. This decontamination procedure could allow reinsertion of an infected implant when peri-prosthetic infection occurs early after breast augmentation or breast reconstruction and when a salvage procedure is indicated.

No Level Assigned This journal requires that authors assign a level of evidence to each submission to which Evidence-Based Medicine rankings are applicable. This excludes Review Articles, Book Reviews, and manuscripts 
that concern Basic Science, Animal Studies, Cadaver Studies, and Experimental Studies. For a full description of these Evidence-Based Medicine ratings, please refer to the Table of Contents or the online Instructions to Authors www.springer.com/00266.

Keywords Infected implant - Implant salvage · Breast augmentation $\cdot$ Breast reconstruction

\section{Introduction}

Infection is the leading cause of morbidity after breast implantation and complicates $2.0-2.5 \%$ of interventions in most case series. Two-thirds of infections develop in the acute post-operative period $[1,2]$.

Organisms most often involved in breast prosthesis infection are Staphylococci (68.2\%), usually S. aureus or S. epidermidis [3]. These organisms belong to normal skin flora and can colonize mammary ducts [4-8]. After incision of the skin and transection of the mammary ducts, the breast pocket is rapidly colonised. A brief skin contact can be sufficient for prosthesis contamination. It has been demonstrated that organisms can adhere to breast implants after only 2 min of contact [9].

Management of early breast prosthesis infection after breast augmentation or breast reconstruction is contentious. Opinions remain divided regarding either a two-stage replacement procedure, with removal and delayed reinsertion after 6 months, which remains the gold standard $[10,11]$, or a more aggressive, one-stage management, which involves thorough cleaning of the pocket after prosthesis removal and insertion of a new implant $[1,12-17]$.

In the context of one-stage surgical management, the aim of this study was to determine if bacteria and their biofilms could be removed from a recently colonized breast prosthesis, without damaging the implant. In other words, when salvage is indicated, is reinsertion of the same prosthesis after decontamination feasible?

\section{Materials and Methods}

This experimental study was performed following the ethical guidelines of the University of Liège. A total of 112 shell samples of silicone breast prostheses, smooth (SPSS) and textured (TPSS), were colonized by S. epidermidis (SE) or S. aureus (SA) strains, all able to produce biofilms. The study protocol is summarized in Fig. 1.

\section{Organisms}

Two strains of Staphylococcus originally isolated from infected intra-vascular catheters cultured in the medical microbiology laboratory were used: Staphylococcus epidermidis F0038576 and Staphylococcus aureus F0041616. These strains were inoculated onto sheep's blood Columbia agar and incubated overnight at $35^{\circ} \mathrm{C}$. Colonies were harvested and suspended in sterile saline solution. The turbidity of each suspension was adjusted to 0.5 MacFarland barium sulfate standard, equivalent to $10^{8}$ colonyforming units (CFU) per ml. To assess the number of CFU/ $\mathrm{ml}$, a series consisting of tenfold dilutions of the bacterial suspension was quantitatively inoculated onto sheep's blood Columbia agar. Numbers of colonies were assessed after overnight incubation at $35^{\circ} \mathrm{C}$.

\section{Breast Implants}

Two types of breast implants were studied: Smooth surface, cohesive gel filled prosthesis (Mentor ${ }^{\circledR}$ Corporation Santa Barbara, CA, USA) and Siltex ${ }^{\circledR}$ textured surface, cohesive gel filled prosthesis (Mentor ${ }^{\circledR}$ Corporation Santa Barbara, CA, USA). In sterile conditions, 56 rectangular samples of $2.5 \times 1 \mathrm{~cm}^{2}$ were punched from the prosthesis shells. Any remaining silicone gel on the internal side of the samples was removed with sterile gauze. A total of 112 samples were tailored. Each one was placed in a single sterile Petri dish to constitute 4 groups of 20 samples: SPSS/SE (group I), SPSS/SA (group II), TPSS/SE (group III), TPSS/SE (group IV). In another group - group SEM-, 16 samples were used for documentation with scanning electron microscopy (SEM). The remaining 16 samples were used to test the limits of detection in the sterility test (group TEST).

\section{Contamination and Colonization}

Each sterile shell sample of groups I-IV and group SEM was immersed in $10 \mathrm{ml}$ of Columbia broth and further contaminated with $100 \mu \mathrm{l}$ of a $10^{8} \mathrm{CFU} / \mathrm{ml}$ suspension of Staphylococcus aureus or Staphylococcus epidermidis. For each type of prosthesis, 24 segments were contaminated per species of Staphylococcus. These cultures were incubated for 15 days at $35{ }^{\circ} \mathrm{C}$ (Fig. 1). After 15 days, all the samples were extracted from the contaminated culture broth. Contamination of the sample was attested by the presence of bacteria fixed on the wall of the tube and turbidity of the culture medium. 


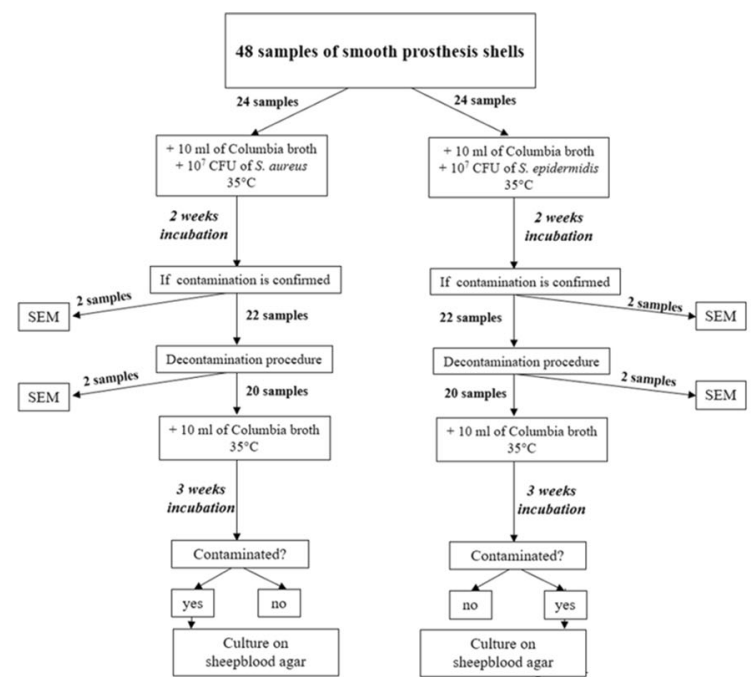

Fig. 1 Rectangular samples $\left(482.5 \times 1 \mathrm{~cm}^{2}\right)$ were punched in the smooth and in the textured prosthesis shells. A total of 96 samples were tailored. For each type of prosthesis, 24 samples were

\section{Decontamination}

After 2 weeks, the colonized segments of groups I-IV and 8 colonized segments of group SEM were taken from the culture tube and were submitted to the decontamination procedure (Fig. 1). They were immersed in a $100 \%$ povidone-iodine (Iso-Betadine ${ }^{\circledR}$ Dermique, PVP-I 10\% solution aqueuse Purdue Frederick, Stamford, CT, USA) bath and both sides were cleaned with a sterile smooth brush. This procedure took $<1 \mathrm{~min}$ for each sample. Then, the samples were rinsed with a saline solution and dried with sterile gauze. Acute peri-prosthetic infection occurs, on average, 10-12 days (minimum 6 days and maximum 6 weeks) after surgery [2]. This is why our decontamination procedure was carried out on day 15 , corresponding to the time of a potential salvage procedure in surgical practice.

\section{Sterility Testing}

To detect surviving microorganisms after decontamination, each sample was immersed in $10 \mathrm{ml}$ of Columbia broth and incubated at $35{ }^{\circ} \mathrm{C}$ for 3 weeks. Cultures were examined for growth every day. Growth was assessed by the presence of turbidity. If a positive culture occurred, it was inoculated onto sheep's blood Columbia agar and incubated at $35{ }^{\circ} \mathrm{C}$ to check if the contaminant was the initial contaminating species, S. aureus or S. epidermidis (Fig. 1).

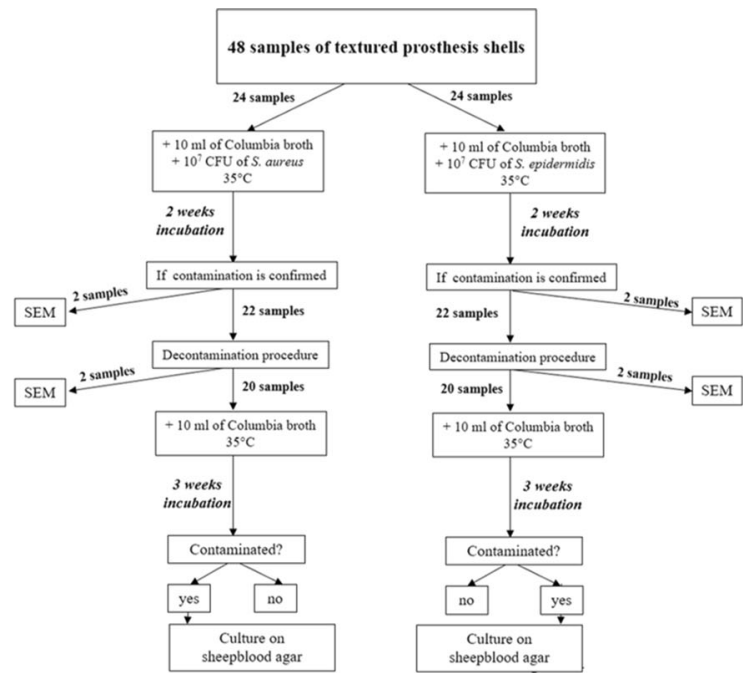

contaminated per species of Staphylococcus and submitted to the sterility test. $C F U$ colony-forming units, SEM scanning electron microscopy

\section{Limits of Detection in the Sterility Test (Group TEST)}

To determine the lower limits of recovery and detection of surviving microorganisms in small numbers from contaminated samples of prosthetic materials $(1 \times 2.5 \mathrm{~cm}), 4$ segments per type of prosthetic material and per species of Staphylococcus were contaminated on their outer surface with $100 \mu \mathrm{l}$ of bacterial suspensions containing, respectively, $1,10,10^{2}$ and $10^{3}$ bacteria. These samples were left at room temperature for $1 \mathrm{~h}$ in an aseptic environment. Each segment was then examined for surviving microorganisms, as outlined above (Fig. 2).

\section{Scanning Electron Microscopy (Group SEM)}

Submission to the scanning electron microscopy (SEM) analysis was performed after 2 weeks contamination (subgroup SEM+) and after decontamination (subgroup SEM-). For each strain and for each type of prosthetic material, 2 colonized (SEM+) and 2 'washed' (SEM-) samples were submitted for SEM analysis (Fig. 1). All specimens were immersed in a $0.1 \mathrm{M}$ cacodylate buffer solution. A fixing step was applied by soaking the samples for $45 \mathrm{~min}$ in a glutaraldehyde solution $(2.5 \mathrm{vol} \%$ in $0.1 \mathrm{M}$ cacodylate buffer) at room temperature. The samples were then rinsed with water and lyophilized. A Pt conductive coating was deposited by plasma sputtering (Balzers). The samples were mounted on aluminium holders and observed at $15 \mathrm{kV}$ in a FEG-ESEM (XL30 from FEI company) under high vacuum. Each specimen was viewed at a magnification of 100-1000. 


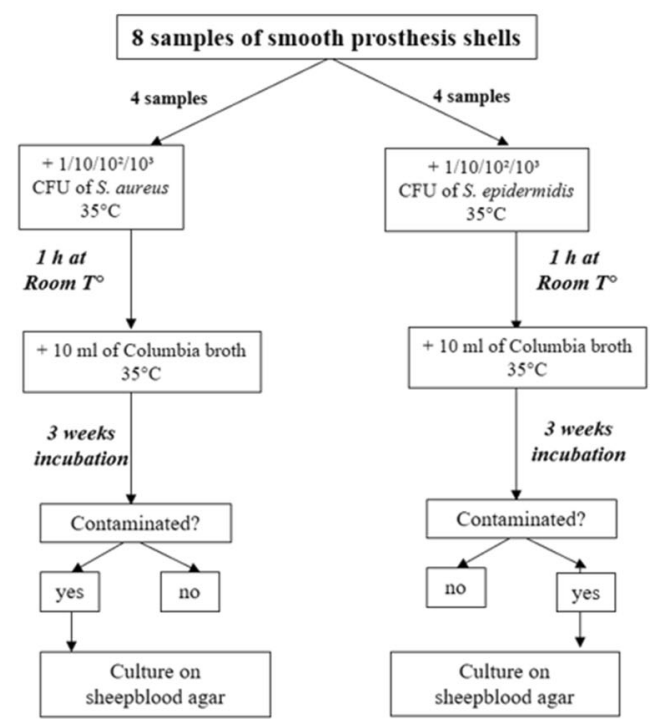

Fig. 2 Eight rectangular samples $\left(2.5 \times 1 \mathrm{~cm}^{2}\right)$ were punched in the smooth and in the textured prosthesis shells. A total of 16 samples were tailored. For each type of prosthesis and per species of

\section{Data Analysis}

In this study, 112 shell samples of silicone breast prostheses, smooth and textured, were colonized by S. epidermidis or S. aureus strains, capable of producing biofilms. Data analysis was performed using the SPSS program (SPSS 22.0).

\section{Results}

\section{Limits of Detection in the Sterility Test (Group TEST)}

The lower limits of recovery of surviving microorganisms from quantitatively contaminated segments of prosthetic materials were $1 \mathrm{CFU}$ for the smooth prosthetic material either for S. aureus or S. epidermidis and $10 \mathrm{CFU}$ for the textured material. These results are not statistically different from microbiological cultures of serial 10th dilutions used for contamination.

\section{Groups I-IV}

Two weeks after contamination, colonization of the silicone samples was confirmed for all 20 samples in group I (SPSS/SE), II (SPSS/SA), III (TPSS/SE), and IV (TPSS/ SE) (Fig. 3). Three weeks after the decontamination procedure, the four sets of cultures corresponding to each contaminating organism per type of prosthetic material were negative. These results are summarized in Table 1.

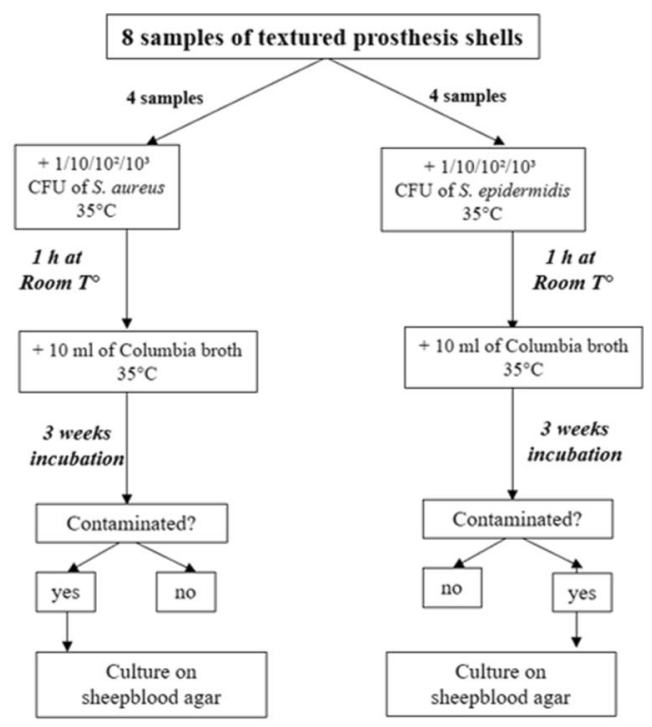

Staphylococcus, 4 samples were used to determine the limits of detection in the sterility tests. CFU colony-forming units, SEM scanning electron microscopy

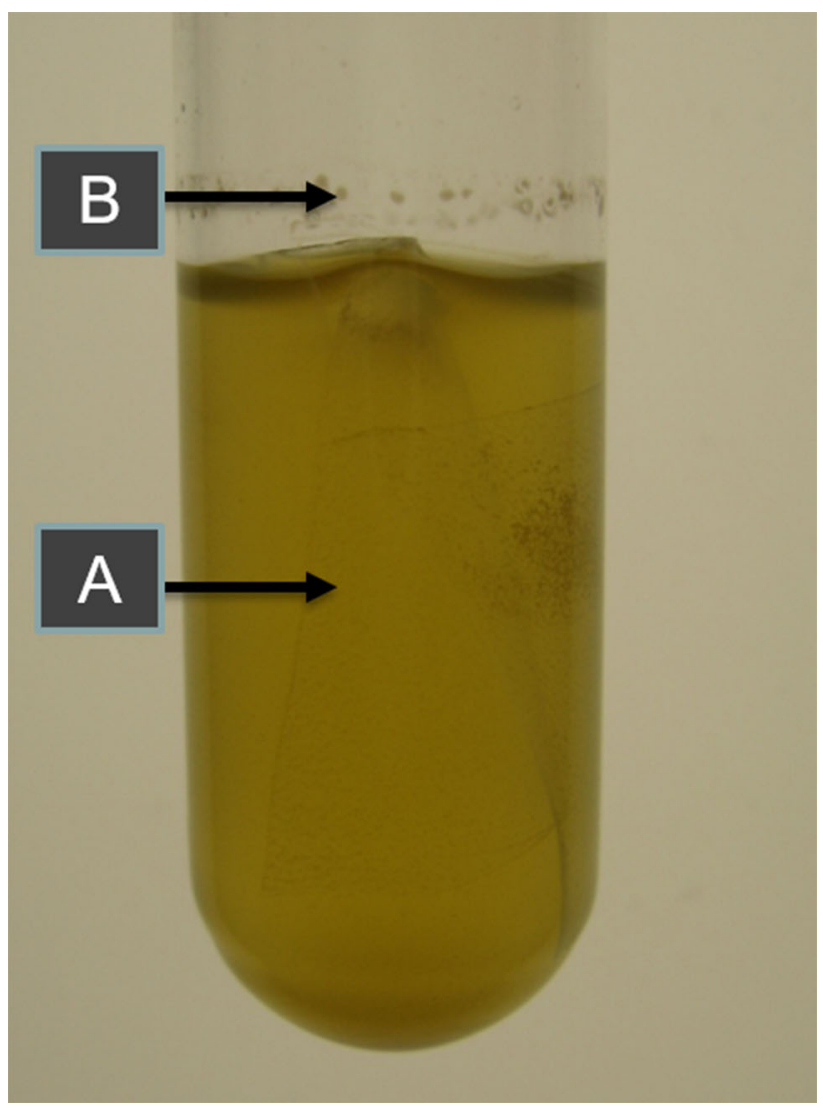

Fig. 3 Contamination of the sample was attested by turbidity of the culture medium (a) and the presence of bacteria fixed on the wall of the tube (b) 
Table 1 Number of positive tests and limits of detection in the sterility tests in the four sets of cultures corresponding to each contaminating organism per type of prosthetic material 3 weeks after decontamination procedure

\begin{tabular}{|c|c|c|c|}
\hline $\begin{array}{l}\text { Type of prosthetic } \\
\text { material }\end{array}$ & $\begin{array}{l}\text { Contaminating } \\
\text { bacteria }\end{array}$ & $\begin{array}{l}\text { Number of positive tests after } \\
\text { decontamination }\end{array}$ & $\begin{array}{l}\text { Limits of detection in the sterility tests } \\
\text { (CFU) }\end{array}$ \\
\hline \multirow[t]{2}{*}{ Smooth } & S. aureus & $0 / 20$ & 1 \\
\hline & S. epidermidis & $0 / 20$ & 1 \\
\hline \multirow[t]{2}{*}{ Textured } & S. aиeus & $0 / 20$ & 10 \\
\hline & S. epidermidis & $0 / 20$ & 10 \\
\hline
\end{tabular}

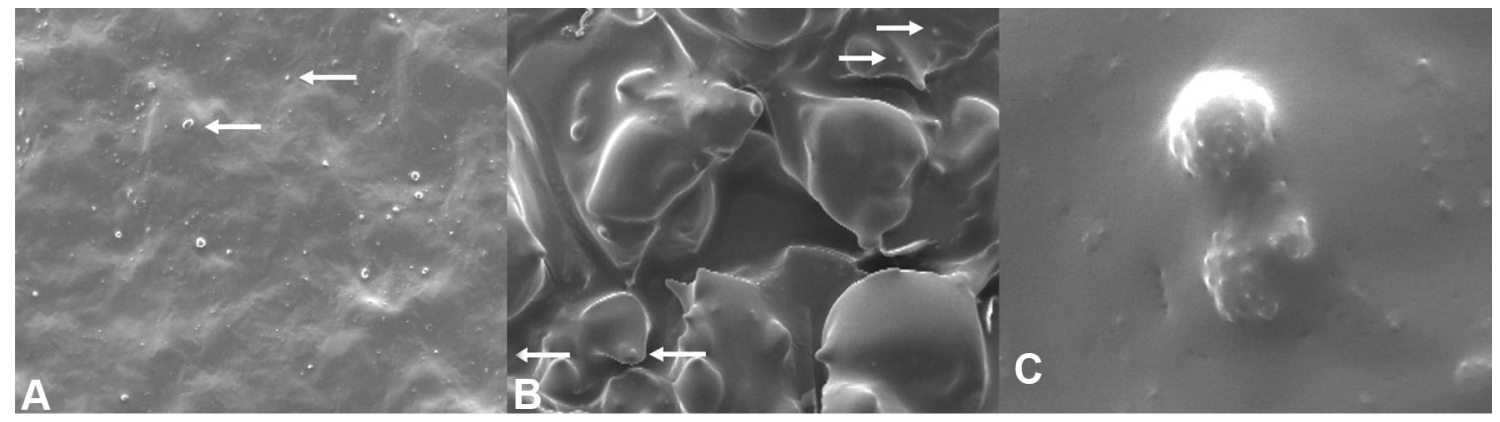

Fig. 4 Scanning electron microscopy (SEM) shows bacteria colonies fixed on the outer surface of the prosthesis shells. a Smooth surface prosthesis covered by Staphyloccus aureus colonies, located with white arrows (Original magnification $\times 100$ ), b textured surface

\section{Group SEM}

After contamination, SEM analysis of the outer surface of the samples in group SEM+, textured or smooth, showed bacterial colonies gathered in groups of 10 bacteria, coated in bioslime (Fig. 4). Bacterial concentration on the outer surface of the implants appeared to be lower in the textured group. After decontamination, no remaining bacteria could be seen on the outer surface of the samples in group SEM-. The structure of the outer surface of the shell was not affected by the decontamination procedure.

\section{Discussion}

In this study, 112 shell samples of silicone breast prostheses, both smooth and textured, were colonized by $S$. epidermidis or $S$. aureus strains capable of producing biofilms. Fifteen days after contamination, all the samples were colonized. SEM images demonstrated the presence of bacteria and biofilm fixed on the shells. Sterility tests performed after decontamination in all groups of implants remained negative for all samples. After cleaning, SEM did not reveal any organisms and there was no alteration in the outer structure of the shell surface.

The organisms most commonly implicated in breast prosthesis infection are Staphylococci $(68.2 \%)$, usually $S$. prosthesis covered by Staphylococcus epidermidis colonies, located with white arrows (Original magnification $\times 100$ ), $\mathbf{c}$ high magnification of two Staphylococcus aureus colonies (Original magnification $\times 2000)$

aureus or S. epidermidis [3]. Staphylococci produce an exopolysaccharide extracellular material commonly referred to as slime. This slime production is enhanced in the presence of a foreign body [18]. Even in individuals with excellent cellular and humoral immunity, biofilm infections are rarely eradicated by the host defence mechanisms [19]. Antimicrobial therapy usually fails to kill bacteria within the biofilm [20]. After conservative treatment alone, biofilm and entrapped bacteria may continue to grow and cause subclinical infection, which may play a major role in capsular contracture [21,22], and may act as a reservoir for acute exacerbations. Indeed, some of the "sessile" organisms may be released by the biofilm and become "planktonic", initiating a new acute peri-prosthetic infection. The only way definitively to eradicate infection is to surgically eliminate the organisms fixed on the surface of the foreign body.

In this context, the first step of our study was to create a model of an infected implant: Two strains of Staphylococci originally isolated from infected intra-vascular catheters and two frequently used types of prostheses (smooth and textured) were used to mimic the clinical scenario. After 2 weeks, all samples were colonized by groups of bacteria (S. Epidermis or S. aureus). SEM images showed colonies of about ten cells, embedded in their bioslime (Fig. 4c). It has been shown that the physical characteristics of a surface (rough or smooth) influence bacterial adhesion to only 
a minor extent [23]. In our study, colonies seemed to be less dense in the textured group but this was not statistically significant and SEM was only used to obtain images of the colonization of the prosthesis samples.

The second step was to try to disunite the bioslime, and the embedded cells, from the implants. Once a biofilm has formed and the exopolysaccharide matrix has been secreted by the sessile cells, the resultant structure is highly viscoelastic and behaves rather like rubber. Donlan and Costerton have clearly demonstrated that biofilms formed in low-shear environments (as in our situation) have a low tensile strength and break easily, but biofilms formed at higher shear (i.e., rapidly flowing milieus) are remarkably strong and resistant to mechanical breakage [24]. To break the biofilm, the colonized shell samples were immersed in a povidone-iodine bath and gently cleaned with a smooth brush [2]. The mechanical action of the smooth brush is necessary to detach the fixed colonies. Moreover, the antiseptic action of Betadine against bacteria like $S$. epidermidis and their biofilm has been well documented in the literature [25]. The integrity of the samples after this combined procedure was evidenced in this study. The minor disruptions induced by a smooth brush on the prosthesis were insignificant compared to the tests performed at the factory (Mentor ${ }^{\circledR}$ Corporation Santa Barbara, Calif.). Moreover, the decision to use Betadine was also affirmed by the experimental study of Zambacos and Nguyen [26], who studied the effects of different concentrations of povidone-iodine $(0.01-10 \%)$ on silicone shells and found no structural modification [26]. In other words, a brief contact of the implant with Betadine, during the decontamination procedure, and followed by copious saline solution irrigation, has no effect on the long-term integrity of the shell of the implant. This is also confirmed by the SEM analysis of the SEM - group, which demonstrates integrity of the samples. Finally, this combination of antiseptic and manual dissolution was effective because 3 weeks after the decontamination procedure, the four sets of cultures corresponding to each contaminating organism per type of prosthetic material were negative and analysis of the surface of the implants in SEM+ group did not reveal any residual bacteria. It should be emphasized that when a breast implant is inserted or reinserted, it is impossible to exclude bacterial contamination of the pocket and/or the implant, even if all necessary precautions are taken (decontamination of the implant, disinfection of the pocket, minimal operating time, no skin contact with the implant, and so on). The key is to reduce the bacterial load to such a point that the remaining bacteria can be eliminated or controlled by the host.

Despite the encouraging results obtained in this study, some limitations are to be noted: (1) Only two strains of Staphylococcus were used in this study. Samples contaminated with a higher number, or several strains of bacteria [27-30] may better mirror the numerous clinical situations with different time frames and hospital contaminants. Moreover, the bacterial content of the breast implant pocket could not be studied. However, Pittet et al. [2] clearly demonstrated that $S$. aureus or S. epidermidis are the most common causative organisms in breast prosthesis infections. (2) Additionally, the limits of detection in the sterility tests were slightly different in both groups, but as previously mentioned, this difference is not significant from the microbiological point of view. In the smooth prosthesis group, $1 \mathrm{CFU}$ is the limit, versus $10 \mathrm{CFU}$ in the textured prosthesis group. This means that even if the sterility tests are negative 3 weeks after the decontamination procedure, we cannot exclude that one bacterium might be left on the textured shell group and fewer than 10 bacteria on the smooth shell. Indeed, bacteria subject to harsh conditions may "hibernate" until conditions are more favorable for proliferation. (3) Finally, the low number of samples in the SEM groups meant they could only serve as photographic documentation. A higher number of samples may have better defined the link between the type of implant and its influence on bacterial adhesion.

\section{Conclusion}

Breast prostheses recently contaminated with strains of Staphylococci, frequently involved in peri-prosthetic breast implant infection and capable of producing biofilms, can be efficiently decontaminated by the procedure used in this study. Our decontamination procedure did not alter surface structure. This decontamination procedure could allow reinsertion of an infected implant when peri-prosthetic infection occurs early after breast augmentation or breast reconstruction and when a salvage procedure is indicated.

\section{Compliance with Ethical Standards}

Conflict of interest The authors declare that they have no conflicts of interest to disclose.

\section{References}

1. Araco A, Gravante G, Araco F, Delogu D, Cervelli V, Walgenbach K (2007) Infections of breast implants in aesthetic breast augmentations: a single-center review of 3,002 patients. Aesthet Plast Surg 31:325-329

2. Pittet B, Montandon D, Pittet D (2005) Infection in breast implants. Lancet Infect Dis 5:94-106

3. Brown SL, Hefflin B, Woo EK, Parmentier CM (2001) Infections related to breast implants reported to the Food and Drug Administration, 1977-1997. J Long Term Eff Med Implants 11:1-12 
4. Barbieri R, Pesce M, Franchelli S, Baldelli I, De Maria A, Marchese A (2015) Phenotypic and genotypic characterization of Staphylococci causing breast peri-implant infections in oncologic patients. BMC Microbiol 15:26

5. Courtiss EH, Goldwyn RM, Anastasi GW (1979) The fate of breast implants with infection around them. Plast Reconstr Surg 63:812-816

6. Henderson JR, Kandola S, Hignett SP, Teasdale RL, Topps AR, Pennick M, Hwang M, Barnes N, Kirwan CC (2017) Infection prophylaxis for breast implant surgery: could we do better? Eplasty 14:17-19

7. Lohmeyer JA, Alawadi M, Bergmann PA, Schmauss D, Wittig KS (2017) Adequate antibiotic prophylaxis and therapy of periprosthetic infections of the breast-review of 468 consecutive implant removals. Handchir Mikrochir Plast Chir 49:91-102

8. Hart A, Desai K, Yoo J, Losken A (2017) Incidence of methicillin-resistant Staphylococcus aureus (MRSA) carrier status in patients undergoing post-mastectomy breast reconstruction. Aesthet Surg J 37:35-43

9. Sanger JR, Sheth NK, Franson TR (1990) Adherence of microorganisms to breast prostheses: an in vitro study. Ann Plast Surg 22:337-342

10. Gabriel SE, Woods JE, O'Fallon WM, Beard CM, Kurland LT, Melton LJ III (1997) Complications leading to surgery after breast implantation. N Engl J Med 336:677-682

11. Darouiche RO (2004) Treatment of infections associated with surgical implants. N Engl J Med 350:1422-1429

12. Chun JK, Chulman MR (2007) The infected breast prosthesis after mastectomy reconstruction: successful salvage of nine implants in eight consecutive patients. Plast Reconstr Surg 120:581-589

13. Planas J, Car bonell A, Planas J (1995) Salvaging the exposed mammary prosthesis. Aesthet Plast Surg 19:535-540

14. Sherif RD, Ingargiola M, Sanati-Mehrizy P, Torina PJ, Harmaty MA (2017) Use of antibiotic beads to salvage infected breast implants. J Plast Reconstr Aesthet Surg Epub May 22

15. Reish RG, Damjanovic B, Austen WG Jr, Winograd J, Liao EC, Cetrulo CL, Balkin DM, Colwell AS (2013) Infection following implant-based reconstruction in 1952 consecutive breast reconstructions: salvage rates and predictors of success. Plast Reconstr Surg 131:1223-1230

16. Agarwal S, Ettinger RE, Kung TA, Kozlow JH, Brown DL (2017) Cohort study of immediate implant exchange during acute infection in the setting of breast reconstruction. J Plast Reconstr Aesthet Surg 70:865-870
17. De Lorenzi C (2005) Successful treatment of acute periprosthetic breast infection with curettage, pulse lavage, and immediate device exchange. Aesthet Plast Surg 29:400-403

18. Christensen GD, Simpson WA, Bisno AL, Beachey EH (1983) Experimental foreign body infections in mice challenged with slime-producing Staphylococcus Epidermidis. Infect Immun 40:407-410

19. Davies DG, Geesey GG (1995) Regulation of the alginate biosynthesis gene algC in Pseudomonas aeruginosa during biofilm development in continuous culture. Appl Environ Microbiol 61:860-865

20. Costerton JW, Stewart PS, Greenberg EP (1999) Bacterial biofilms: a common cause of persistant infections. Science 284:1318-1322

21. Burkhardt BR (2004) Detection of subclinical infections in significant breast implant capsules. Plast Reconstr Surg 113:448

22. Netscher DT (2004) Subclinical infection as a possible cause of significant breast capsules. Plast Reconstr Surg 113:2229-2230

23. Costerton JW, Lewandowski Z, Caldwell DE, Korber DR, Lappin-Scott HM (1995) Microbial biofilms. Annu Rev Microbiol 49:711-745

24. Donlan RM, Costerton JW (2002) Biofilms: survival mechanisms of clinically relevant microorganisms. Clin Microbiol Rev 15:167-193

25. Kunisada T, Yamada K, Oda S, Hara O (1997) Investigation on the efficacy of povidone-iodine against antiseptic-resistant species. Dermatology 195:14-18

26. Zambacos GJ, Nguyen D, Morris RJ (2004) Effect of povidone iodine on silicone gel breast implants in vitro: implications for clinical practice. Plast Reconstr Surg 114:706-710

27. Bernardi C, Saccomanno F (1998) Late Klebsiella pneumoniae infection following breast augmentation: case report. Aesthet Plast Surg 22:222-224

28. Marchese A, Barbieri R, Pesce M, Franchelli S, De Maria A (2016) Breast implant infection due to Paenibacillus residui in a cancer patient. Clin Microbiol Infect 22:743-744

29. Brickman M, Parsa AA, Parsa FD (2005) Mycobacterium cheloneae infection after breast augmentation. Aesthet Plast Surg 29:116-118

30. Lenne A, Defourny L, Lafosse A, Martin M, Vandercam B, Berlière M, Lengelé B, Rodriguez-Villalobos H (2016) Breast prosthesis infection and pets: a case report and review of the literature. Int J Surg Case Rep 22:98-100 